\title{
Terminalidade, morte e luto na pandemia de COVID-19: demandas psicológicas emergentes e implicações práticas
}

\author{
Terminality, death and grief in the COVID-19 Pandemic: \\ emerging psychological demands and practical implications
}

\author{
Maria Aparecida CREPALDI ${ }^{1}$ (D) 0000-0002-5892-7330 \\ Beatriz SCHMIDT² (iD) 0000-0003-2907-2297 \\ Débora da Silva NOAL ${ }^{3}$ (DD 0000-0003-3970-6783 \\ Simone Dill Azeredo BOLZE' ${ }^{1}$ (D) 0000-0002-8715-8913 \\ Letícia Macedo GABARRA4 (iD) 0000-0002-0312-2295
}

\section{Resumo}

A doença causada pelo novo coronavírus (COVID-19) tem sido considerada uma grave crise sob o ponto de vista epidemiológico e, também, psicológico. Além das perdas em massa em curto espaço de tempo, as dificuldades para realização de rituais de despedida entre pessoas na iminência da morte e seus familiares, bem como de rituais funerários, podem dificultar a experiência de luto. O objetivo deste estudo é sistematizar conhecimentos sobre os processos de terminalidade, morte e luto no contexto da pandemia de COVID-19. Por meio de revisão narrativa da literatura, foram sumarizadas experiências relatadas em diferentes países durante a pandemia. Apresentam-se características das demandas psicológicas emergentes e implicações para a prática profissional do psicólogo. Considerando que expressões de afeto,

\section{$\operatorname{rat}$}

${ }^{1}$ Universidade Federal de Santa Catarina, Centro de Filosofia e Ciências Humanas, Programa de Pós-Graduação em Psicologia. Florianópolis, SC, Brasil.

${ }^{2}$ Universidade Federal do Rio Grande, Instituto de Ciências Humanas e da Informação, Programa de Pós-Graduação em Psicologia. Av. Itália, km 8, Carreiros, 96203-900, Rio Grande, RS, Brasil. Correspondência para/Correspondence to: B. SCHMIDT. E-mail: <psi. beatriz@gmail.com>.

${ }^{3}$ Fundação Oswaldo Cruz, Escola de Governo, Centro de Estudos e Pesquisas em Emergências e Desastres em Saúde. Brasília, DF, Brasil.

${ }^{4}$ Universidade Federal de Santa Catarina, Hospital Universitário Polydoro Ernani de São Thiago, Unidade de Terapia Intensiva Adulto. Florianópolis, SC, Brasil.

$\boldsymbol{\nabla} \mathbf{v} \boldsymbol{v}$

Como citar este artigo/How to cite this article

Crepaldi, M. A., Schmidt, B., Noal, D. S., Bolze, S. D. A., \& Gabarra, L. M. (2020). Terminalidade, morte e luto na pandemia de COVID-19: demandas psicológicas emergentes e implicações práticas. Estudos de Psicologia (Campinas), 37, e200090. https://doi.org/ $10.1590 / 1982-0275202037 \mathrm{e} 200090$ 
condolências e espiritualidade sofrem alterações nesse cenário, discute-se a importância de potencializar formas alternativas e respeitosas para ritualização dos processos vividos, o que parece essencial para ressignificar perdas e enfrentar desafios durante e após a vigência da pandemia.

Palavras-chave: Cuidados paliativos na terminalidade da vida; Infecções por coronavírus; Pandemias; Saúde mental.

\begin{abstract}
The disease caused by the new coronavirus (COVID-19) has been regarded as a serious crisis from both an epidemiological and a psychological point of view. In addition to the mass loss of life in a short space of time, the difficulties involved in the performance of farewell rituals between people on the verge of death and their families, not to mention funeral rituals, may exacerbate the grieving experience. The aim of this study is to systematize knowledge about the processes of terminality, death and grief in the context of the COVID-19 pandemic. Through narrative review of the literature, experiences reported in different countries during the pandemic were summarized. Characteristics of the emerging psychological demands and implications for the psychologist's professional practice are presented. Considering that expressions of affection, condolence and spirituality undergo changes in this scenario, the importance of enhancing alternative and respectful ways to ritualize the processes experienced is discussed, which appears to be essential for the reframing of loss and to face the challenges presented during and after the pandemic.
\end{abstract}

Keywords: Hospice care; Coronavirus infections; Pandemics; Mental health.

A doença causada pelo novo coronavírus Coronavirus Disease 2019 (COVID-19), foi inicialmente reportada na China, em dezembro de 2019 (Costantini, Sleeman, Peruselli, \& Higginson, 2020; Ho, Chee, \& Ho, 2020). Frente ao rápido crescimento do número de casos confirmados e de mortes em decorrência da COVID-19 em diferentes países, a Organização Mundial da Saúde passou a caracterizá-la como uma pandemia, a partir de 11 de março de 2020 (World Health Organization, 2020a). Estudos clínicos revelaram que sintomas da COVID-19 comumente envolvem fadiga, tosse, febre e dificuldades respiratórias (Costantini et al., 2020). Embora grande parte dos infectados (i.e., aproximadamente 80\%) apresente sintomas leves ou moderados (Bajwah et al., 2020), um significativo número de casos requer internação hospitalar e, até mesmo, tratamento em unidade de terapia intensiva (Ferguson et al., 2020). A preocupação com a capacidade dos sistemas de saúde para atender à demanda crescente, incluindo a necessidade de leitos hospitalares e respiradores, tem levado à proposição de medidas para conter a rápida escalada da doença (Walker et al., 2020), tais como fechamento de universidades e escolas, restrições a viagens, isolamento de casos suspeitos e distanciamento social para toda a população (C. Wang et al., 2020; Ferguson et al., 2020). Apesar dos impactos socioeconômicos derivados dessas medidas, estima-se que seja necessário mantê-las em algum grau até que vacinas ou tratamentos efetivos estejam disponíveis, no sentido de salvar o máximo possível de vidas humanas (Walker et al., 2020).

Estudos têm revelado que tanto a pandemia propriamente dita quanto as medidas adotadas para contê-la parecem impactar a saúde mental, aumentando o risco para surgimento de sintomas de estresse, ansiedade e depressão, o que vem sendo identificado na população geral (C. Wang et al., 2020) e em profissionais da saúde (Zhang et al., 2020). Nesse sentido, a COVID-19 pode ser considerada uma crise sob o ponto de vista epidemiológico e, também, psicológico (Weir, 2020a), dadas as alterações cognitivas, emocionais e comportamentais que tendem a ser experienciadas nesse período (Enumo, Weide, Vicentini, Araujo, \& Machado, 2020). Em linhas gerais, as pandemias se associam a perdas em massa, tanto de vidas humanas (Scanlon \& McMahon, 2011), quanto de rotinas, conexões sociais face a face e estabilidade financeira (Taylor, 2019; Weir, 2020a). Por conta da COVID-19, muitas pessoas têm vivenciado mudanças rápidas em seu dia a dia e precisam lidar com o futuro imprevisível (Weaver \& Wiener, 2020). Outras tantas foram infectadas ou mesmo perderam alguém da sua rede socioafetiva em decorrência da doença (Arango, 2020). 
Ademais, o processo de terminalidade e morte também tem sido afetado durante a pandemia (Fundação Oswaldo Cruz [Fiocruz], 2020a; Social Science in Humanitarian Action Platform [SSHAP], 2020). Em função das medidas de distanciamento social adotadas em alguns países (Ferguson et al., 2020; Walker et al., 2020), pessoas hospitalizadas mantêm contato com seus familiares por meio de telefones celulares ou tablets, quando podem utilizá-los (Arango, 2020; Ingravallo, 2020). Doações desses equipamentos a hospitais têm sido realizadas, com o intuito de favorecer os processos comunicacionais (SSHAP, 2020). Profissionais da saúde que trabalham na chamada "linha de frente" (i.e., em interação direta com os infectados), com destaque a enfermeiros e médicos, mesmo sobrecarregados com a intensa demanda de cuidados físicos, têm buscado oferecer apoio emocional às pessoas hospitalizadas, o que tende a ser exaustivo (Ingravallo, 2020). Nesse contexto, torna-se mais complexa a realização de rituais de despedida entre doentes na iminência da morte e seus familiares, bem como de rituais funerários, o que pode dificultar a experiência de luto (Eisma, Boelen, \& Lenferink, 2020; Fiocruz, 2020a). Além disso, a ocorrência de múltiplos casos de infecção (Bajwah et al., 2020) e óbito em uma mesma família gera lutos sequenciais, trazendo desafios adicionais à forma de se adaptar e lidar com as perdas (Wallace, Wladkowski, Gibson, \& White, 2020).

Contudo, ainda quando não há perdas concretas (e.g., morte de um familiar), as pessoas podem experienciar sofrimento, por empatia àquelas mais diretamente afetadas e por sensibilização à instabilidade social gerada pela pandemia (Weir, 2020a). Assim, entende-se que as implicações psicológicas desencadeadas pela COVID-19 podem ser mais prevalentes e duradouras que o próprio acometimento pela doença (Ornell, Schuch, Sordi, \& Kessler, 2020; Schmidt, Crepaldi, Bolze, Neiva-Silva, \& Demenech, 2020). Isso sugere a relevância de intervenções psicológicas tanto durante quanto após a vigência da pandemia, considerando também a chamada "segunda onda" (Shojaei \& Masoumi, 2020), com destaque às demandas relacionadas aos processos de terminalidade, morte e luto (Taylor, 2019; Weir, 2020a).

Diante do exposto, o objetivo do presente estudo é sistematizar conhecimentos sobre os processos de terminalidade, morte e luto no contexto da pandemia de COVID-19, buscando caracterizar demandas psicológicas emergentes e discutir implicações para a prática. Para tanto, foram sumarizadas experiências que vêm sendo relatadas em diferentes países durante a pandemia, bem como discutidas possíveis adaptações às intervenções tradicionalmente utilizadas por psicólogos em processos de terminalidade, morte e luto, considerando as restrições impostas frente à COVID-19. Realizou-se revisão narrativa da literatura, visto que tal estratégia se mostra adequada para sistematização de conhecimentos sobre tópicos ainda pouco explorados, oferecendo subsídios para o desenvolvimento de novas modalidades de intervenção (Ferrari, 2015). A revisão narrativa da literatura permite também a integração de estudos sobre uma mesma temática produzidos com base em diferentes abordagens metodológicas (Pautasso, 2020), o que favorece as reflexões sobre problemáticas cujas pesquisas são recentes e adotam diferentes delineamentos, como é o caso da pandemia de COVID-19 (Schmidt et al., 2020).

Para reduzir possíveis vieses na seleção dos materiais (Ferrari, 2015), foram realizadas buscas por meio de diferentes bases de dados e portais de pesquisa (e.g., Medical Publications [PubMed], ScienceDirect, Google Scholar e Scientific Electronic Library Online [SciELO]), utilizando descritores como "end-of-life", "palliative care", "death", "grief", "bereavement", "coronavirus" e "COVID-19". Esse processo ocorreu em diferentes dias do mês de abril de 2020. Também foram acessados sites de organizações ligadas à área da saúde e à Psicologia, no Brasil e no mundo, em busca dos desenvolvimentos mais recentes sobre os processos de terminalidade, morte e luto no contexto da pandemia de COVID-19. Com base nas vivências profissionais e de pesquisa das autoras do presente estudo em Psicologia da Saúde, Psicologia Hospitalar, bem como Psicologia na Gestão Integral de Riscos e de Desastres, refletiu-se sobre possíveis desafios e potencialidades para a atuação de psicólogos nesse cenário. Os achados da revisão narrativa da literatura são apresentados nas seções: Terminalidade, morte e luto: demandas psicológicas emergentes; e, Implicações para a prática de psicólogos diante das perdas na pandemia de COVID-19. 


\section{Terminalidade, morte e luto: demandas psicológicas emergentes}

Pandemias costumam acarretar mortes em massa em um curto espaço de tempo, o que traz implicações psicológicas diversas (Taylor, 2019). No caso da COVID-19, em particular, algumas medidas adotadas para conter a rápida escalada do número de infectados, incluindo restrições a viagens e distanciamento social (C. Wang et al., 2020; Ferguson et al., 2020), dificultam interações face a face entre enfermos e membros da sua rede socioafetiva (Ingravallo, 2020; Pattison, 2020). Essas interações face a face são consideradas importantes nos chamados "rituais de despedida", isto é, processos de despedida realizados entre pessoas na iminência da morte e seus familiares (Lisbôa \& Crepaldi, 2003). Os rituais de despedida acontecem por meio de incentivo à comunicação familiar, definição de questões não resolvidas, compartilhamento de bons momentos vividos juntos, agradecimentos e pedidos de perdão, revelando-se promotores de qualidade de morte para os doentes e de qualidade de vida para os familiares (Lisbôa \& Crepaldi, 2003; Schmidt, Gabarra, \& Gonçalves, 2011). Ademais, os rituais de despedida tendem a ser organizadores, vindo a favorecer a resolução do luto (Fiocruz, 2020a).

Tanto a comunicação verbal quanto a não verbal se mostram essenciais nos rituais de despedida; a comunicação não verbal, especificamente, parece importante em situações em que as palavras são insuficientes para externalizar o que se deseja ou, ainda, não podem ser ditas (Lisbôa \& Crepaldi, 2003). A pandemia de COVID-19, portanto, impõe desafios adicionais aos rituais de despedida nos casos de terminalidade. O fato de muitas pessoas na iminência da morte estarem isoladas, sem a possibilidade de estabelecer interações face a face, pode dificultar as conversações no final da vida (Pattison, 2020; S. Wang, Teo, Yee, \& Chai, 2020; Weir, 2020b). Adicionalmente, mais de um membro da família pode estar infectado e, até mesmo, hospitalizado (Bajwah et al., 2020). Porém, quando disponíveis, recursos como smartphones ou computadores vêm sendo utilizados, de forma a possibilitar a manutenção de contato com a rede socioafetiva, por meio de telefonemas, mensagens de texto, áudio e vídeo (Arango, 2020; Fiocruz, 2020a; Ingravallo, 2020). Apesar disso, nesses casos há poucas oportunidades para a comunicação não verbal, e mesmo a comunicação verbal pode ser prejudicada, sobretudo quando as pessoas na iminência da morte estão entubadas ou sedadas (Pattison, 2020).

Nesse sentido, profissionais da linha de frente têm se dedicado à humanização dos atendimentos (SSHAP, 2020), com destaque aos cuidados paliativos, buscando controlar sintomas e aliviar sofrimento (Fiocruz, 2020b). Além de opioides, ansiolíticos vêm sendo utilizados (Bajwah et al., 2020; Kunz \& Minder, 2020), visto que pacientes gravemente enfermos podem se mostrar angustiados, tanto pelas dificuldades respiratórias, quanto pelo medo de que sua situação se agrave rapidamente e eles venham a morrer isolados (Bajwah et al., 2020). Destaca-se a oferta de apoio psicológico às famílias, ainda que de forma remota, pelas restrições quanto à presença de visitantes e acompanhantes adotadas em hospitais de diferentes países, e.g.: Espanha (Arango, 2020); Itália (Costantini et al., 2020). Esse apoio psicológico é fundamental, pois a família costuma ser afetada duplamente, pelo agravamento do quadro de saúde do doente e pelo medo de que outros familiares estejam infectados (Fiocruz, 2020b). Apesar de consideradas prioridades nos cuidados paliativos (Weaver \& Wiener, 2020), as necessidades espirituais nem sempre podem ser atendidas no contexto da pandemia, também em função das restrições para interações face a face (Costantini et al., 2020).

Os óbitos por COVID-19 têm ocorrido não somente nos hospitais, mas também nos domicílios (Ingravallo, 2020; Kunz \& Minder, 2020). Em alguns casos, a pessoa infectada é acompanhada por profissionais da saúde e, após explicação compreensível, cuidadosa e realística sobre a gravidade da doença e o mau prognóstico, ela pode tomar a decisão de permanecer em casa, o que deve ser discutido também com seus familiares, para posterior planejamento de cuidados paliativos no ambiente doméstico (Kunz \& Minder, 2020). Por outro lado, os óbitos nos domicílios também podem ocorrer por impossibilidade de acesso aos serviços de saúde, sobretudo pela indisponibilidade de leitos hospitalares (Zibell, 2020). Ademais, há registros de colapso no 
sistema funerário (SSHAP, 2020), demandando que as famílias permaneçam por um longo período com a pessoa que faleceu em casa, até a remoção do corpo (Zibell, 2020), ou mesmo exigindo a abertura de valas comuns, para atender ao aumento repentino no número de sepultamentos (Albuquerque, 2020).

Diferentemente de outros eventos em que também ocorrem mortes em massa (e.g., desastres naturais e acidentes aéreos), rituais funerários que podem favorecer o processo de despedida e a elaboração de sentido para a perda, como velórios e enterros, são proibidos ou realizados com restrições durante pandemias, dado que reunir pessoas aumenta as chances de contágio (Scanlon \& McMahon, 2011), especialmente ao considerar que nessas situações costuma haver proximidade física, apertos de mãos e abraços. Procedimentos de tanatopraxia (e.g., limpeza e preparação do corpo para homenagens fúnebres, o que tem um significado importante para muitas culturas) não são recomendáveis, devido ao alto risco de transmissão póstuma da COVID-19 (SSHAP, 2020). O cadáver deve ser acomodado em caixão a ser lacrado antes da entrega à família (Ministério da Saúde, 2020). Tais mudanças tendem a tornar ainda mais desafiador o processo de luto, sobretudo quando os familiares consideram que o falecido não recebeu o ritual funerário que merecia (Ingravallo, 2020), ou mesmo quando não houve a oportunidade de serem confortados e oferecerem conforto às pessoas próximas, visto que o apoio social auxilia a lidar com as perdas e seguir em frente (Wallace et al., 2020; Weir, 2020a). Esse cenário não é novo, pois a realização de rituais funerários também foi restrita em outras pandemias, como a de influenza H1N1 (Taylor, 2019) e a de Ebola (Victor \& Ahmed, 2019). Contudo, isso dificulta práticas culturais e religiosas socialmente prescritas de manejo e permanência durante algum tempo próximo ao corpo para despedida (Victor \& Ahmed, 2019). Da mesma forma, pode provocar a sensação de negligência e tratamento desumano no final da vida (Taylor, 2019), aumentando o risco para problemas de saúde mental nos sobreviventes após a crise (Victor \& Ahmed, 2019; Wallace et al., 2020).

Na pandemia de COVID-19, entretanto, nota-se uma particularidade, já que em algumas localidades rituais funerários têm sido transmitidos ao vivo ou gravados para serem reproduzidos posteriormente e.g.: Estados Unidos (Hernández \& Berman, 2020); Singapura (S. Wang et al., 2020). Embora esses recursos atualmente disponíveis não substituam os rituais funerários tradicionalmente adotados pelas comunidades, é possível que eles auxiliem as pessoas a se despedir dos que falecem e a se apoiar mutuamente, ainda que de forma virtual, repercutindo na dimensão social da morte e do morrer (Ingravallo, 2020). Não obstante, nem todos se beneficiam desses recursos, tanto pela falta de acesso à internet, smartphones ou computadores, quanto pelas dificuldades para utilizar tais equipamentos, o que costuma ocorrer particularmente entre idosos no Brasil (Schmidt et al., 2020).

Outro aspecto frequente no contexto de pandemias é a ocorrência de adoecimento e óbito de diferentes pessoas em um mesmo núcleo familiar (Bajwah et al., 2020), o que traz estressores adicionais aos processos de despedida e à adaptação às perdas (Wallace et al., 2020). As repercussões negativas geradas nesses casos podem ser potencializadas, a depender da fase do ciclo de vida e das funções desempenhadas na família pela pessoa que faleceu (Schmidt et al., 2011). Em linhas gerais, o óbito de pessoas mais jovens costuma ser especialmente traumático no contexto de pandemias, em particular quando se trata de crianças e adolescentes, ou mesmo de adultos que desempenhavam funções de cuidadores nas famílias (Taylor, 2019). Embora a taxa de mortalidade em decorrência da COVID-19 tenda a aumentar com a idade, sendo a maioria dos óbitos entre pessoas com mais de 60 anos (Lloyd-Sherlock, Ebrahim, Geffen, \& McKee, 2020), mortes entre pessoas mais jovens também têm sido reportadas, principalmente quando há comorbidades, tais como hipertensão, diabetes e cardiopatia, que acabam por exacerbar os sintomas da infecção pelo novo coronavírus (Walker et al., 2020).

Ainda que crianças e adolescentes tenham uma taxa de mortalidade mais baixa por COVID-19 em comparação a pessoas com mais idade, eles permanecem vulneráveis às implicações psicossociais da pandemia sobre seu desenvolvimento e suas relações familiares e comunitárias (Weaver \& Wiener, 2020). Na China, por 
exemplo, destacou-se a importância da atenção às crianças cujos pais ou cuidadores morreram em decorrência da COVID-19 e que, por conseguinte, precisaram ser encaminhadas a instituições de acolhimento, pois o medo e o sofrimento desencadeados pela perda das figuras de referência aumentam o risco para problemas de saúde mental (Liu, Bao, Huang, \& Lu, 2020). Na Espanha, por outro lado, em algumas famílias o óbito dos pais idosos deixou desassistidos os filhos adultos com transtorno mental severo, os quais não tinham condições para lidar com suas necessidades básicas, revelando componentes sociais dessa grave crise de saúde (Arango, 2020).

Em linhas gerais, dadas as múltiplas perdas, principalmente de pessoas da rede socioafetiva, considera-se esperado o luto no contexto de pandemias (Taylor, 2019; Weir, 2020a). O luto consiste em um processo normativo de adaptação às perdas, abrangendo emoções, cognições, sensações físicas e mudanças comportamentais (Wallace et al., 2020; Worden, 2018). Quando essas perdas envolvem pessoas da rede socioafetiva, algumas tarefas são essenciais para elaboração do luto, a saber: aceitação da realidade da perda, pois pode emergir a sensação de que a morte não ocorreu; reconhecimento do sofrimento que a perda acarretou, sem utilizar subterfúgios, visto que evitar ou suprimir a dor tende a prolongá-la; adaptação ao contexto de vida sem a presença da pessoa falecida, o que demanda assumir funções que ela desempenhava anteriormente na família; e, reposicionamento emocional da pessoa falecida, ou seja, organização de um espaço emocional para lembrar dela, de modo que se possa dar continuidade à vida (Worden, 2018).

Ao final da vigência da pandemia de COVID-19, é possível que muitas pessoas consigam elaborar o luto e se adaptar relativamente bem às transformações (Weir, 2020a), ao passo que outras experienciarão luto complicado (Wallace et al., 2020). O luto complicado, por sua vez, envolve a intensificação do sofrimento, sem progressão para resolução ao longo do tempo, de forma que a pessoa se sente sobrecarregada e passa a apresentar comportamentos desadaptativos que a prejudicam na vida diária (Worden, 2018). Dentre os sinais e sintomas de luto complicado, destacam-se: pensamentos invasivos, recorrentes e persistentes sobre a pessoa que morreu; tristeza intensa; afastamento de outras relações interpessoais; e, percepção de falta de sentido na vida (Wallace et al., 2020).

A complexidade do contexto da pandemia de COVID-19 pode impactar o processo de luto de diferentes formas (Bajwah et al., 2020; Wallace et al., 2020). Em primeiro lugar, destaca-se que o luto antecipatório (i.e., experienciado quando há perspectiva de morte, o qual costuma favorecer a preparação emocional para a perda (Schmidt et al., 2011), tende a ser afetado, pois em alguns casos a situação se agrava rapidamente e o paciente vai a óbito (Bajwah et al., 2020). Ademais, o local e a condição em que a morte ocorreu também trazem implicações; e.g., se foi no hospital, com o doente isolado e sem a realização de ritual de despedida, pode haver maiores chances de seus familiares experienciarem luto complicado (Pattison, 2020; Wallace et al., 2020). Outros fatores de risco consistem em: perda de mais de uma pessoa próxima (Worden, 2018); fragilidade de apoio da rede socioafetiva, pelas medidas de distanciamento adotadas para conter a escalada da doença (Wallace et al., 2020); não realização de ritual funerário em conformidade com as práticas culturais e religiosas socialmente prescritas (Victor \& Ahmed, 2019); e, sentimento de culpa que os sobreviventes podem experienciar quando acreditam que foram os responsáveis por infectar a pessoa falecida (Taylor, 2019).

\section{Implicações para a prática de psicólogos diante das perdas na pandemia de COVID-19}

Como visto, a pandemia de COVID-19 tem o potencial para afetar as experiências de terminalidade, morte e luto. Assim, intervenções alinhadas às demandas emergentes nesse contexto são necessárias (Wallace et al., 2020; Weir, 2020a). Dentre os principais desafios do processo de terminalidade, destaca-se o isolamento experienciado em muitos casos, o que dificulta as conversações no final da vida (Pattison, 2020; 
SSHAP, 2020; Weir, 2020b). Nesse sentido, tem sido incentivado o contato entre pessoas na iminência da morte e seus familiares por meio de telefones celulares ou tablets, quando as interações face a face não são possíveis (Arango, 2020; Ingravallo, 2020).

No contexto hospitalar, o psicólogo pode identificar os pacientes com alto risco de morte, para explorar as compreensões sobre a gravidade da doença, o desejo e as condições emocionais, tanto por parte do enfermo quanto das pessoas afetivamente mais próximas, para realização de ritual de despedida; essa abordagem prévia é fundamental, visando à preservação dos envolvidos (Lisbôa \& Crepaldi, 2003; Schmidt et al., 2011). Crianças e adolescentes também podem ser acolhidos e incluídos nesse processo (Schmidt et al., 2011), por meio da criação de um espaço seguro e sensível para expressão de emoções, conforme seu estágio desenvolvimental, buscando reconhecer a morte como parte do ciclo de vida (Weaver \& Wiener, 2020). Se houver recursos disponíveis, interesse por parte dos envolvidos e a situação permitir (e.g., o paciente tem consciência preservada e se comunica, verbal ou não-verbalmente), pode-se realizar videoconferência (Fiocruz, 2020c; S. Wang et al., 2020). Sugere-se que essa reunião seja acompanhada pelo psicólogo, no sentido de oferecer apoio e auxiliar no manejo de situações potencialmente desafiadoras (Schmidt et al., 2011). Após o término da videoconferência é recomendável a escuta individual a cada um dos envolvidos, para suporte e encaminhamentos necessários (S. Wang et al., 2020).

Alternativamente, sugere-se a realização de teleconferência, o envio de cartas ou mesmo de objetos que representem a ligação emocional entre o enfermo e os membros da sua rede socioafetiva, para serem mantidos junto ao leito ou no caixão, como símbolo da conexão contínua entre eles (S. Wang et al., 2020). Se o paciente estiver inconsciente, a família pode ser incentivada a encaminhar mensagens de áudio para reprodução à beira do leito; nesse caso, o psicólogo deve inicialmente informar ao doente sobre o procedimento que será realizado, justificar os motivos para ausência da visita, reproduzir o áudio e, na sequência, dar retorno à família (Fiocruz, 2020c). Para pacientes que não estejam inconscientes, mas tenham poucas condições para comunicação verbal, mensagens de áudio também podem ser utilizadas. Para todos os procedimentos anteriormente descritos, destaca-se a importância de registro em prontuário (Fiocruz, 2020c; S. Wang et al., 2020), bem como a consideração de questões éticas e legais sobre uso de imagens e informações, sendo recomendável a assinatura de Termo de Responsabilidade no momento da admissão no hospital (Fiocruz, 2020c).

É importante lembrar que as recomendações técnico-científicas para contenção da COVID-19 precisam ser incorporadas às estratégias de cuidado das equipes de psicologia, visando garantir a biossegurança dos envolvidos (World Health Organization, 2020b) e, ao mesmo tempo, potencializar formas alternativas e respeitosas de ritualizar os processos vividos (Fiocruz, 2020a). O contato face a face entre o psicólogo e o paciente em isolamento hospitalar pode ser restrito na vigência da pandemia, com o apoio emocional sendo realizado sobretudo por enfermeiros e médicos (Schmidt et al., 2020). Nesses casos, além do acompanhamento remoto ao enfermo e à família, o psicólogo também pode oferecer suporte e orientação aos profissionais da linha de frente (Schmidt et al., 2020; SSHAP, 2020), inclusive nos procedimentos relativos ao ritual de despedida.

Rituais funerários, considerados organizadores do processo de despedida e importantes para elaboração do luto, necessitam de reconfiguração durante pandemias (Scanlon \& McMahon, 2011; Taylor, 2019). De acordo com as orientações sanitárias vigentes, os falecidos em decorrência da COVID-19 devem ser cremados ou alocados em caixão lacrado (Ministério da Saúde, 2020), o que impede a aproximação ou a visualização do corpo. As famílias devem ser informadas de maneira cuidadosa e sensível sobre os procedimentos necessários; elas precisam ter a oportunidade para esclarecer dúvidas e tomar decisões relativas ao falecido, conforme apropriado (SSHAP, 2020). 
Quando possível, recomenda-se a realização de estratégias presenciais de despedida adaptadas às restrições do período, bem como ao contexto sociocultural e familiar, ou mesmo estratégias remotas de despedida (Fiocruz, 2020a; Meyer, 2016; SSHAP, 2020; Worden, 2018). Dentre as estratégias presenciais de despedida, destacam-se: inserir foto da pessoa falecida no caixão ou no espaço de velório, estimular que sejam proferidas mensagens verbais e escritas, bem como cantadas ou reproduzidas músicas consideradas significativas para aquele que morreu e para os membros da sua rede socioafetiva (Fiocruz, 2020a). Quanto às estratégias remotas de despedida, sugere-se a realização de rituais individuais, como acender uma vela em uma janela (SSHAP, 2020), e rituais coletivos, envolvendo incentivo à rede socioafetiva para expressar seus sentimentos por meio de telefonemas, cartas, mensagens de texto e áudio (Fiocruz, 2020a). Salientase também a criação de memoriais online, em que familiares, amigos e outras pessoas manifestam suas condolências e compartilham pensamentos sobre o falecido (Worden, 2018).

A expressão de sentimentos de luto nas redes sociais é um fenômeno relativamente recente, caracterizando-se como alternativa para lembrar e homenagear o falecido, com a garantia de que essa memória fique em local seguro e possa ser atualizada de forma interativa (Meyer, 2016). O Facebook, em particular, permite a transformação de contas de usuários que morreram em memorial; essa página pode ser utilizada para anunciar informações sobre o óbito ou o funeral (Worden, 2018), postar lembranças (e.g., fotos, vídeos e outros conteúdos), de modo a tornar público o processo de luto, o que favorece as manifestações de apoio e a conexão emocional, sobretudo quando o distanciamento não permite o contato físico (Meyer, 2016).

Rituais alternativos estão ocorrendo em diferentes países, para aliviar a dor das perdas em decorrência da COVID-19: em Madri (Espanha), o governo local propôs um minuto de silêncio diariamente e as bandeiras são hasteadas a meio mastro; na Itália, policiais saúdam veículos transportando falecidos; na Holanda, famílias têm solicitado às pessoas da rede socioafetiva que enviem cartões postais, cartas e fotos do falecido, para serem apresentadas em uma cerimônia posterior (SSHAP, 2020). Rituais alternativos relacionados à espiritualidade também podem ser incentivados, o que inclui celebrações religiosas virtuais, tais como cultos, missas e velórios (Fiocruz, 2020a; Hernández \& Berman, 2020). A espiritualidade tende a ser um recurso importante para muitas famílias, podendo contribuir no enfrentamento aos desafios da vida, na adaptação e na resiliência às perdas (Walsh, 2016). Portanto, a espiritualidade costuma favorecer a maneira de lidar com situações adversas, como o luto, de modo que pode ser fortalecida nesse contexto (Biondo, Ferraz, Silva, \& Yarid, 2017). Assim, nos casos em que a família menciona ter uma fé, estabelecida anteriormente à pandemia, é possível recomendar o contato virtual com líderes religiosos de referência (SSHAP, 2020), visando apoiar os enlutados em sua jornada espiritual na busca de maior significado e conexão (Walsh, 2016). Deve-se ter o cuidado de incentivar as famílias a realizar rituais alternativos sem, contudo, pressioná-las, tendo em vista que não há procedimentos certos ou errados (SSHAP, 2020).

Embora essas expressões de condolências, afeto e espiritualidade não substituam os rituais funerários tradicionalmente adotados pelas comunidades (Ingravallo, 2020), é possível que auxiliem na resolução do luto, à medida que oferecem oportunidades emocionais e cognitivas para lidar com a perda (Wallace et al., 2020). Ademais, dado que a pandemia de COVID-19 interrompeu as experiências usuais de luto, gerando medo quanto ao presente e incertezas quanto ao futuro, acompanhar a família na adaptação após a morte é importante (Wallace et al., 2020; Weir, 2020a). No que diz respeito aos hospitais, recomenda-se que um dos membros da equipe de saúde (e.g., psicólogo) avalie o luto pós-funeral, por videoconferência ou teleconferência, realizando encaminhamentos necessários quando houver indicativos de risco para luto complicado (S. Wang et al., 2020).

Destaca-se a importância de acompanhar as repercussões dos processos de terminalidade, morte e luto em série sobre a saúde mental da equipe de saúde (Arango, 2020; Fiocruz, 2020b), considerando que os óbitos podem ocorrer entre pacientes atendidos e colegas de trabalho durante a vigência de pandemias (Scanlon \& McMahon, 2011; Taylor, 2019). Além disso, salienta-se também atenção às repercussões psicológicas negativas 
experienciadas por profissionais que trabalham em funerárias, os quais descrevem se sentir sobrecarregados e angustiados, em particular após encontrar familiares que tentam entregar objetos a serem colocados junto do falecido, com o caixão já lacrado (SSHAP, 2020).

Em linhas gerais, o trabalho do psicólogo junto aos profissionais que atuam durante a fase de resposta e recuperação da pandemia deve considerar especialmente a promoção de autocuidado, para auxiliá-los a lidar com as adversidades desse período, o que inclui orientá-los sobre: fazer pausas e se desconectar da pandemia (Wallace et al., 2020); sintomas psicológicos que podem emergir nesse contexto (e.g., insônia, estresse, ansiedade e depressão; Zhang et al., 2020); estratégias de enfrentamento (e.g., manejo de estresse; Taylor, 2019). Sugere-se também a escuta empática e a oferta de apoio psicológico, bem como a prestação ou o encaminhamento para intervenções psicológicas mais específicas, quando necessário (Schmidt et al., 2020). O psicólogo também pode auxiliar na estruturação do processo de trabalho desses profissionais, de forma a evitar a sobrecarga e o rápido desgaste; parte dessa estratégia pode consistir na alternância dos profissionais da linha de frente e na diminuição da carga horária (Fiocruz, 2020c).

\section{Considerações Finais}

No presente estudo, foram sistematizamos conhecimentos sobre os processos de terminalidade, morte e luto no contexto da pandemia de COVID-19. Em síntese, buscou-se caracterizar demandas psicológicas emergentes e discutir implicações para a prática. Foi destacada a importância do reconhecimento de que os processos de terminalidade, morte e luto são experienciados de forma singular, não havendo uma sequência estanque ou normatizadora, tampouco rigidez nos rituais que favorecem a despedida e a elaboração de sentido para as perdas em tempos de pandemia. Potencialidades e recursos familiares devem ser enfatizados pelo psicólogo, ao invés das perdas unicamente, na perspectiva do fortalecimento das redes socioafetivas, do compromisso e da solidariedade entre as pessoas, o que parece essencial para enfrentar desafios tanto durante quanto após a vigência da pandemia.

Por fim, salientamos também a importância do cuidado à saúde mental do próprio psicólogo, frente às adversidades vivenciadas diante dessa grave emergência de saúde pública. Dado que o presente estudo se referiu a uma revisão narrativa da literatura, que sumarizou sobretudo experiências relatadas internacionalmente, recomendamos a realização de pesquisas sobre os processos de terminalidade, morte e luto no contexto da pandemia de COVID-19 no Brasil, de modo que seja possível conhecer como esses processos estão sendo experienciados e propor intervenções alinhadas às especificidades das demandas do cenário nacional.

\section{Contribuição}

M. A. CREPALDI e B. SCHMIDT contribuíram na concepção, desenho, análise e discussão dos resultados, na revisão do manuscrito e aprovação da versão final. D. S. NOAL e S. D. A. BOLZE contribuíram na análise e discussão dos resultados, na revisão do manuscrito e aprovação da versão final. L. M. GABARRA contribuiu na revisão do manuscrito e aprovação da versão final.

\section{Referências}

Albuquerque, A. N. (2020, 21 de abril). Enterros triplicam, e cemitério de Manaus abre valas comuns para vítimas do coronavírus. Folha de S. Paulo. Recuperado de https://www1.folha.uol.com.br/cotidiano/2020/04/enterros-triplicam-ecemiterio-de-manaus-abre-valas-comuns-para-vitimas-do-coronavirus.shtml 
Arango, C. (2020). Lessons learned from the coronavirus health crisis in Madrid, Spain: how COVID-19 has changed our lives in the last two weeks [Ahead of Print]. Biological Psychiatry. https://dx.doi.org/10.1016/j.biopsych.2020.04.003

Bajwah, S., Wilcock, A., Towers, R., Costantini, M., Bausewein, C., Simon, S. T., ... Higginson, I. J. (2020). Managing the supportive care needs of those affected by COVID-19. European Respiratory Journal, 55, 2000815. https://dx.doi. org/10.1183/13993003.00815-2020

Biondo, C. S., Ferraz, M. O. A., Silva, M. L. M., \& Yarid, S. D. (2017). Espiritualidade nos serviços de urgência e emergência. Revista Bioética, 25(3), 596-602. https://dx.doi.org/10.1590/1983-80422017253216

Costantini, M., Sleeman, K. E., Peruselli, C., \& Higginson, I. J. (2020). Response and role of palliative care during the COVID-19 pandemic: a national telephone survey of hospices in Italy [Ahead of Print]. Palliative Medicine. https://dx.doi.org/10.1101/2020.03. 18.20038448

Crepaldi, M. A., Schmidt, B., Noal, D. S., Bolze, S. D. A., \& Gabarra, L. M. (2020). Terminalidade, morte e luto na pandemia de COVID-19: demandas psicológicas emergentes e implicações práticas. Scielo Preprints. Versão 1. https:// doi.org/10.1590/SciELOPreprints.491

Eisma, M. C., Boelen, P. A., \& Lenferink, L. I. (2020). Prolonged grief disorder following the Coronavirus (COVID-19) pandemic. Psychiatry Research, 288, 113031. https://dx.doi.org/10.1016/j.psychres.2020.113031

Enumo, S. R. F., Weide, J. N., Vicentini, E. C. C., Araujo, M. F., \& Machado, W. L. (2020). Enfrentando o estresse em tempos de pandemia: proposição de uma cartilha. Estudos de Psicologia (Campinas), 37, e200065. http://dx.doi. org/10.1590/1982-0

Ferguson, N., Laydon, D., Nedjati Gilani, G., Imai, N., Ainslie, K., ... Ghani, A. (2020). Report 9: impact of Non-Pharmaceutical Interventions (NPIS) to reduce COVID19 mortality and healthcare demand. London: Imperial College. Retrieved from http://hdl.handle.net/10044/1/77482

Ferrari, R. (2015). Writing narrative style literature reviews. Medical Writing, 24(4), 230-235. https://dx.doi.org/10.117 9/2047480615z.000000000329

Fundação Oswaldo Cruz. (2020a). Saúde mental e atenção psicossocial na pandemia COVID-19: processo de luto no contexto da COVID-19. Rio de Janeiro: Autor. Recuperado de https://www.fiocruzbrasilia.fiocruz.br/wp-content/ uploads/2020/04/sa\%c3\%bade-mental-e-aten\%c3\%a7\%c3\%a3o-psicossocial-na-pandemia-covid-19-processo-deluto-no-contexto-da-covid-19.pdf

Fundação Oswaldo Cruz. (2020b). Saúde mental e atenção psicossocial na pandemia COVID-19: cuidados paliativos. Rio de Janeiro: Autor. Recuperado de https://wnww.fiocruzbrasilia.fiocruz.br/wp-content/uploads/2020/04/sa\%c3\%bade-emental-e-aten\%c3\%a7\%c3\%a3o-psicossocial-na-pandemia-covid-19-cuidados-paliativos-orienta\%c3\%a7\%c3\%b5es-aosprofissionais-de-sa\%c3\%bade.pdf

Fundação Oswaldo Cruz. (2020c). Saúde mental e atenção psicossocial na pandemia COVID-19: orientações às/aos psicólogas/os hospitalares. Rio de Janeiro: Autor. Recuperado de https://www.fiocruzbrasilia.fiocruz.br/wp-content/ uploads/2020/04/cartilha_psicologos_hospitalares.pdf

Hernández, A. R., \& Berman, M. (2020, March 23). Grief amid the pandemic: live-streamed funerals, canceled services and mourning left 'unfinished'. The Washington Post. Retrieved from https://www.washingtonpost.com/national/griefamid-the-pandemic-live-streamed-funerals-canceled-services-and-mourning-left-unfinished/2020/03/23/9201e996-6bdf-11eaabef-020f086a3fab_story.html

Ho, C. S., Chee, C. Y., \& Ho, R. C. (2020). Mental health strategies to combat the psychological impact of COVID-19 beyond paranoia and panic. Annals, Academy of Medicine, Singapore, 49(1), 1-3. Retrieved from http://www.anmm. org.mx/descargas/Ann-Acad-Med-Singapore.pdf

Ingravallo, F. (2020). Death in the era of the COVID-19 pandemic. The Lancet Public Health, 5(5), e258. https://dx.doi. org/10.1016/S2468-2667(20)30079-7

Kunz, R., \& Minder, M. (2020). COVID-19 pandemic: palliative care for elderly and frail patients at home and in residential and nursing homes. Swiss Medical Weekly, 150(w20235), 1-2. https://dx.doi.org/10.4414/smw.2020.20235

Lisbôa, M. L., \& Crepaldi, M. A. (2003). Ritual de despedida em familiares de pacientes com prognóstico reservado. Paidéia, 13(25), 97-109. https://dx.doi.org/10.1590/50103-863X2003000200009

Liu, J. J., Bao, Y., Huang, X., Shi, J., \& Lu, L. (2020). Mental health considerations for children quarantined because of COVID-19. The Lancet Child \& Adolescent Health, 4(5), 347-349. https://dx.doi.org/10.1016/S2352-4642(20)30096-1

Lloyd-Sherlock, P., Ebrahim, S., Geffen, L., \& McKee, M. (2020). Bearing the brunt of covid-19: older people in low and middle income countries. BMJ, 368, m1052. https://dx.doi.org/10.1136/bmj.m1052

Meyer, E. P. (2016). Death in the age of eternity: how Facebook users cope with personal loss (Unpublished master's thesis). Iwoa State University. Retrieved from https://lib.dr.iastate.edu/cgi/viewcontent.cgi?article=6779\&context=etd 
Ministério da Saúde (2020). Manejo de corpos no contexto do novo coronavírus - COVID-19. Brasilia: Autor. Recuperado de https:// www.saude.gov.br/images/pdf/2020/marco/25/manejo-corpos-coronavirus-versao1-25mar20-rev5.pdf

Ornell, F., Schuch, J. B., Sordi, A. O., \& Kessler, F. H. P. (2020). "Pandemic fear" and COVID-19: mental health burden and strategies [Ahead of Print]. Brazilian Journal of Psychiatry. Retrieved from https://www.rbppsychiatry.org.br/ details/943/en-us/-pandemic-fear--and-covid-19--mental-health-burden-and-strategies

Pattison, N. (2020). End-of-life decisions and care in the midst of a global coronavirus (COVID-19) pandemic. Intensive and Critical Care Nursing, 102862, 1-3. https://dx.doi.org/10.1016/j.iccn.2020.102862

Pautasso, M. (2020). The structure and conduct of a narrative literature review. In R. S. Tubbs, S. M. Buerger, M. M. Shoja, A. Arynchyna, \& M. Karl (Eds.), A guide to the scientific career: virtues, communication, research, and academic writing (pp.299-310). Hoboken: Wiley Blackwell.

Scanlon, J., \& McMahon, T. (2011). Dealing with mass death in disasters and pandemics. Disaster Prevention and Management, 20(2), 172-185. https://dx.doi.org/10.1108/09653561111126102

Schmidt, B., Crepaldi, M. A., Bolze, S. D. A., Neiva-Silva, L., \& Demenech, L. M. (2020). Saúde mental e intervenções psicológicas diante da pandemia do novo coronavírus (COVID-19). Estudos de Psicologia (Campinas), 37, e200063. http://dx.doi.org/10.1590/1982-0 275202037e200063

Schmidt, B., Gabarra, L. M., \& Gonçalves, J. R. (2011). Intervenção psicológica em terminalidade e morte: relato de experiência. Paidéia, 21(50), 423-430. https://dx.doi.org/10.1590/\$0103-863X2011000300015

Shojaei, S. F., \& Masoumi, R. (2020). The importance of mental health training for psychologists in COVID-19 outbreak. Middle East Journal of Rehabilitation and Health Studies, 7(2), e102846. https://dx.doi.org/10.5812/mejrh.102846

Social Science in Humanitarian Action Platform. (2020). Key considerations: dying, bereavement and mortuary and funerary practices in the context of COVID-19 (Brief). Retrieved from https://www.ids.ac.uk/publications/key-considerationsdying-bereavement-and-mortuary-and-funerary-practices-in-the-context-of-covid-19-april-2020/

Taylor, S. (2019). The psychology of pandemics: preparing for the next global outbreak of infectious disease. Newcastle upon Tyne: Cambridge Scholars Publishing.

Victor, G. S., \& Ahmed, S. (2019). The importance of culture in managing mental health response to pandemics. In D. Huremovic (Ed.), Psychiatry of pandemics: a mental health response to infection outbreak (pp.55-64). Cham: Springer.

Walker, P. G., Whittaker, C., Watson, O., Baguelin, M., Ainslie, K. E. C., Bhatia, S., ... Ghani, A. C. (2020). The global impact of COVID-19 and strategies for mitigation and suppression. London: Imperial College. Retrieved from https:// unw.imperial.ac.uk/media/imperial-college/medicine/sph/ide/gida-fellowships/mperial-College-COVID19-Global-Impact-26-032020v2.pdf

Wallace, C. L., Wladkowski, S. P., Gibson, A., \& White, P. (2020). Grief during the COVID-19 pandemic: considerations for palliative care providers [Ahead of Print]. Journal of Pain and Symptom Management. https://dx.doi.org/10.1016/j. jpainsymman.2020.04.012

Walsh, F. (2016). A dimensão espiritual da vida familiar. In F. Walsh (Ed.), Processos normativos da familia: diversidade e complexidade (pp.347-371). Porto Alegre: Artmed.

Wang, C., Pan, R., Wan, X., Tan, Y., Xu, L., Ho, C. S., \& Ho, R. C. (2020). Immediate psychological responses and associated factors during the initial stage of the 2019 coronavirus disease (COVID-19) epidemic among the general population in china. International Journal of Environmental Research and Public Health, 17(5), 1729. https://dx. doi.org/10.3390/ ijerph17051729

Wang, S. S., Teo, W. Z., Yee, C. W., \& Chai, Y. W. (2020). Pursuing a good death in the time of COVID-19 [Ahead of Print]. Journal of Palliative Medicine. https://dx.doi.org/10.1089/jpm.2020.0198

Weaver, M. S., \& Wiener, L. (2020). Applying palliative care principles to communicate with children about COVID-19 [Ahead of Print]. Journal of Pain and Symptom Management. https://dx.doi.org/10.1016/j.jpainsymman.2020.03.020

Weir, K. (2020a, April, 1). Grief and COVID-19: mourning our bygone lives. American Psychological Association. Retrieved from https://www.apa.org/news/apa/2020/04/grief-covid-19

Weir, K. (2020b, April 6). Grief and COVID-19: saying goodbye in the age of physical distancing. American Psychological Association. Retrieved from https://www.apa.org/topics/covid-19/grief-distance

World Health Organization. (2020a). WHO Director-General's opening remarks at the media briefing on COVID-19-11 March 2020. Geneve: Author. Retrieved from https://www.who.int/dg/speeches/detail/who-director-general-s-openingremarks-at-the-media-briefing-on-covid-19---11-march-2020 
Word Health Organization. (2020b). Infection prevention and control during health care when novel coronavirus (nCoV) infection is suspected. Geneve: Author. Retrieved from https://www.who.int/publications-detail/infection-prevention-andcontrol-during-health-care-when-novel-coronavirus-(ncov)-infection-is-suspected-20200125

Worden, J. W. (2018). Grief counseling and grief therapy: a handbook for the mental health practitioner. New York: Springer.

Zhang, C., Yang, L., Liu, S., Ma, S., Wang, Y., Cai, Z., ... Zhang, B. (2020). Survey of insomnia and related social psychological factors among medical staffs involved with the 2019 novel coronavirus disease outbreak. Frontiers in Psychiatry, 11, 1-9. https://dx.doi.org/10.3389/fpsyt.2020.00306

Zibell, M. (2020, 1 de abril). Mortos em casa e cadáveres nas ruas: o colapso funerário causado pelo coronavírus no Equador. BBC News Brasil. Recuperado de https://www.bbc.com/portuguese/internacional-52129845

Recebido: maio 4, 2020

Versão final: maio 15, 2020

Aprovado: maio 20, 2020 